\title{
A Crise do Sistema Único de Saúde e a Fuga para o Mercado*
}

\author{
Rosângela Minardi Mitre Cotta ${ }^{1}$ \\ José Norberto Muniz ${ }^{2}$ \\ Fábio Faria Mendes ${ }^{3}$ \\ José Sette Cotta Filho ${ }^{4}$
}

Resumo: Este trabalho discute as conseqüências paradoxais das ações em saúde, pensadas a partir do ideal universalizante igualitário, consubstanciado na Constituição de 1988; bem como os dilemas que hoje se interpõem ao setor público em saúde.

Uma análise da conjuntura atual do relacionamento entre o setor público e o privado em saúde foi desenvolvida tendo como subsídio a investigação empírica, com atenção especial ao conhecimento local.

A técnica amostral utilizada foi a "amostragem por experts". Através deste método, identificam-se os atores mais relevantes para o processo de construção e implantação do Sistema Único de Saúde. Impõe-se, assim, a combinação de conhecimentos estratégicos específicos, derivados de vários atores políticos inseridos direta ou indiretamente no processo de implementação da política de saúde em curso.

Quarenta e nove entrevistas participantes foram realizadas com atores relevantes para o setor saúde, escolhidos em instituições públicas e privadas da saúde. Finalmente, procedeu-se à análise documental e estatística.

Os resultados mostraram que as formas perversas de interação do sistema público de saúde com o mercado, conseqüência da maneira desorganizada de o governo implementar a Reforma Sanitária, apontam para um retrocesso na forma de custeio da saúde no país. Tem-se, de um lado, a obrigatoriedade de contribuição previdenciária e, de outro, o pagamento de seguros privados de saúde, sem, ao final, ter-se a garantia de um atendimento satisfatório.

Institui-se, portanto, na saúde, um jogo do "salve-se quem puder", jogo em que a fuga para o mercado nào representa uma saída total do sistema público, nem garantia de atendimento.

Palavras-Chave: Políticas de Saúde; O SUS e o mercado em saúde; Sistema público e privado em saúde.

1 Professora Assistente de Políticas Públicas em Saúde e de Planejamento e Administração em Saúde do Departamento de Nutrição e Saúde (DNS) da Universidade Federal de Viçosa (UFV).

${ }^{2}$ Professor Titular de Geraçào de Tecnologia, Sociologia do Conhecimento e Metodologia da pesquisa I e II, do Departamento de Economia Rural (DER) da Universidade Federal de Viçosa (UFV).

${ }^{3}$ Professor Assistente de Estado e Políticas Públicas e Sociologia do Departamento de Economia Rural (DER) da Universidade Federal de Viçosa (UFV).

${ }^{4}$ Médico Sanitarista

* Trabalho Apresentado sob a forma de "Comunicação Coordenada", no V Congresso Brasileiro de Saúde Coletiva e V Congresso Paulista de Saúde Pública, de 25 a 29 de agosto, em Águas de Lindóia, SP. 
Summary: The paper discusses the paradoxes of some unanticipated consequences of the actions in the field of collective health, created by the universal egalitarian ideal formulated by the Brazilian Constitution, and also the dilemmas that nowadays are posed to the public health system.

An analysis based on the present hypotheses on the relationship between the public and private health care systems was carried out using an empirical investigation, with special attention for the local knowledge.

The sampling technique used was the "experts sampling method". This method identify the most important actors involved in the process of institutional implantation and management in the realm of the Brazilian Unified Health System (SUS).

Forty-nine participants were interviewed, all of them key actors in both public and private health institutions. Finally, a comprehensive survey of available documents and statistical analyses were undertaken.

The results highlight a perverse interaction of the Unified Health System with the market, as a consequence of the unorganized implementation of the Sanitary Reform, determining serious barriers toward the implementation of a reliable and equitable financial structure of Brazilian Health System. The public social insurance taxes are compulsory in Brazil, but the common citizen pays also private health insurance plans, but remain unsure about the perspectives of receiving satisfactory treatment.

So, in the Brazilian health sector, a game of "rescue yourself, if you can" is going on, in which neither the public system or the market address in fact the complex problems in the domain of medical care.

Keywords: Health policy in Brazil; The Brazilian Unified Health System and the Market; Public and private health.

\section{Introdução}

O presente estudo discute efeitos paradoxais de conseqüências não antecipadas das ações em saúde, inspiradas pelo "ideal universalizante igualitário", consubstanciado no artigo 194, itens I e III, da Constituição Federal de 1988. Pretende-se, assim, tratar dos dilemas que hoje se interpõem ao setor público em saúde.

Este trabalho ocupa-se, portanto, da análise da conjuntura atual do relacionamento entre o setor público e o privado em saúde. No contexto local pesquisado, os dados apontam para a proposição de que, como nas outras esferas da sociedade brasileira, o sistema de saúde se privatiza em conseqüência da forma desorganizada (intencional ou "não") de o governo implantar e implementar as reformas.

Nesse sentido, não se analisa a concepção do programa da Reforma em si, mas a situação em que se encontra, em virtude desta Reforma ocorrer em um contexto geral de privatização. Constata-se, assim, que, no Sistema de Saúde, a privatização ocorre mediante ações que não se estabelecem imediatamente, ou seja, a privatização não requer atividades explícitas do governo que, pelo contrário, num primeiro momento, estrategicamente optou por não tê-las. O que se verifica é que o governo deixou, e continua deixando, todos os espaços possíveis para que os planos de saúde privados em suas diversas modalidades evoluam naturalmente. Institui-se, de acordo com O'Donnell (1993), "um mundo do salve-se quem puder", em que jogar esse jogo reforça as próprias condições em que ele é jogado.

Vencida essa etapa, o governo dá agora um novo passo. Explicitamente, tenta legitimar (inclusive com reformas constitucionais) sua política excludente e privatista de insti- 
tuir um Sistema de Saúde público para os pobres, aqueles que não podem pagar, e um sistema privado para as camadas sociais de melhor poder aquisitivo. Esse fato, em termos práticos, não significará apenas um retrocesso histórico, mas o desmonte do Sistema Único de Saúde (SUS), única e legítima forma de se garantir a saúde como direito.

\section{Metodologia}

Tem-se como subsídio para esta análise, a investigação empírica, teoricamente fundamentada, com atenção especial para o conhecimento local. A referência espacial é a microrregião de saúde de Ponte Nova, localizada na Zona da Mata de Minas Gerais.

Inicialmente, foram realizados estudos exploratórios em alguns municípios e instituições de saúde da região, com o objetivo de obter informações sobre aspectos relevantes para a pesquisa. Além disso, foram obtidos, também, dados de outras fontes, tais como os documentos do Ministério da Saúde, da Secretaria Estadual da Saúde e da Diretoria Regional da Saúde, além da participação em encontros realizados na região e em reuniões de Conselhos Municipais de Saúde. Para complementar o estudo exploratório, realizaram-se entrevistas semi-estruturadas para coleta de informações com os agentes atuantes no contexto de gerenciamento do SUS, principalmente com aqueles ligados à Diretoria Regional e à Secretaria Estadual de Saúde.

Considerando-se os aspectos destacados anteriormente, optou-se pela utilização da amostragem por expert. Mais especificamente, Babbie (1983:178) ressalta que:

"A amostragem por expert é selecionada, baseada no fato de você possuir conbecimentos sobre a populaçâo, os elementos $e$ a natureza dos objetivos de sua pesquisa: em resumo, fundamenta-se no seu julgamento e nos propósitos do estudo a ser realizado."

Por meio desse processo amostral, o pesquisador identifica os experts que poderiam fornecer informações mais adequadas e relevantes para os objetivos do trabalho (Warwick \& Lininger, 1975). Deve-se explicitar que essa estratégia amostral foi também utilizada para selecionar a microrregião de saúde onde seria feita a pesquisa, as instituições e os atores inseridos no contexto de implantação e implementação do SUS em nível local.

A regional de saúde de Ponte Nova é composta por 26 municípios e subdivide-se em duas microrregiões: a de Ponte Nova e a de Viçosa. A microrregião de saúde de Ponte Nova tem um número de municípios superior à de Viçosa (17 e 9, respectivamente) e é maior quanto à população $(214.239 \mathrm{e}$ 106.874 habitantes, respectivamente), quanto ao número de unidades de saúde hospitalares (10 e 6 , respectivamente) e também quanto à diversidade em relação à natureza da gestão municipal de saúde. A microrregião de saúde de Ponte Nova, quando comparada à de Viçosa, apresenta, portanto, maior variabilidade em relação ao objeto em estudo, o que justifica a sua seleção para o desenvolvimento do presente estudo.

Para a realização da pesquisa de campo, selecionaram-se os seguintes municípios: Alvinópolis, Diogo de Vasconcelos, Guaraciaba e Ponte Nova. A amostra é composta por municípios em situações diferentes no que se refere ao porte (grande, médio e pequeno porte), natureza da gestão municipal (gestão parcial, incipiente e ainda não habilitado) e organização dos serviços de saúde (possuem ou não hospital, e outras unidades de saúde). A caracterização desses municípios pode ser visualizada no Quadro 1. 


\begin{tabular}{|l|c|c|c|c|}
\hline Quadro 1 - Caracterização dos Municípios Amostrados da Microrregiāo de Saúde de Ponte Nova-MG. 1995 \\
\hline Municípios & Alvinópolis & Diogo de Vasconcelos & Guaraciaba & Ponte Nova \\
\hline População/Porte do Município & $\begin{array}{c}15.333 \\
\text { Médio porte }\end{array}$ & $\begin{array}{c}4.170 \\
\text { Pequeno porte }\end{array}$ & $\begin{array}{c}10.545 \\
\text { Médio porte }\end{array}$ & $\begin{array}{c}56.688 \\
\text { Grande porte }\end{array}$ \\
\hline Hospitais & 1 & 0 & 1 & 2 \\
\hline Leitos & 60 & - & 35 & 425 \\
\hline Outras unidades de saúde & $\begin{array}{c}4 \text { postos } \\
\text { de saúde }\end{array}$ & $\begin{array}{c}4 \text { postos } \\
\text { de saúde }\end{array}$ & $\begin{array}{c}\text { de saúde } \\
\text { de saúde e es } \\
1 \text { ambulatório } \\
\text { municipal }\end{array}$ \\
\hline $\begin{array}{l}\text { Habilit. NOB./93 - } \\
\text { Natureza da gestão }\end{array}$ & Parcial & Incipiente & Não habilitado & Parcial \\
\hline
\end{tabular}

Fontes: SUS/SOS/SES/DRS-PN/Coordenadoria de Informaçào - Sistema de Controle da Municipalização, 1994. IBGE - Censo Demográfico, 1991.

Foram realizadas entrevistas participantes (49) com atores relevantes para o setor saúde, a partir de instituições tais como: Secretarias Municipais de Saúde, Diretoria Regional de Saúde, Instituições Privadas de Saúde, Instituições Públicas de Saúde, Prefeituras e Conselhos Municipais de Saúde. Realizou-se, ainda, análise documental e estatística.

\section{Resultados e Análise}

Embora haja uma grande distância entre a formalidade da lei e a sua aplicação, especialmente no que se refere ao cumprimento dos princípios e diretrizes do SUS (Jelin, 1993), pode-se considerar que o processo de municipalização da saúde começa a ser implantado, formalmente, na microrregião em estudo, a partir do ano de 1990. A elaboração do primeiro Plano de Saúde e a criação da Secretaria Municipal de Saúde em Ponte Nova marcam o início desse processo. Entretanto, é a partir de 1991 que a Secretaria Municipal de Saúde começa a efetivamente funcionar.

Com a municipalização, inicia-se a fiscalização das contas emitidas pelas instituições privadas conveniadas com o SUS. Em conseqüência, segundo alguns entrevistados, os hospitais perdem gradativamente o poder de utilização dos chamados "mecanismos de compensação", tais como a utilização de múltiplos procedimentos e substituição dos procedimentos que remuneram mal por outros mais lucrativos. Este fato, associado aos constantes atrasos no pagamento pelos serviços prestados e às baixas tabelas pagas pelo SUS, faz com que as instituições privadas encontrem formas alternativas de manter seus lucros. O governo passa a não ser mais um cliente "confiável e lucrativo".

Os administradores hospitalares entrevistados destacam que, a partir de 1991, ocorre o incremento de outros tipos de seguros privados de saúde pelas instituições locais. A insatisfação das "classes médias" da população, do operariado de melhor nível salarial e das próprias empresas com a dificuldade de acesso aos serviços de saúde, assim como a queda da qualidade da assistência médica proporcionada pelo SUS parecem estabelecer as condiçôes favoráveis e impulsionadoras para o fortalecimento das diferentes modalidades de atenção médica supletiva (medicina de grupo, seguros-saúde, planos privados 
próprios, cooperativas médicas e planos de administração). O Quadro 2 apresenta as modalidades de convênios e as percentagens de arrecadações nas instituições de saúde amostradas.

Deve-se ressaltar, entretanto, certas limitações no que se refere às inferências relativas ao incremento de convênios privados de saúde e à diminuição do atendimento do SUS pelas instituições privadas conveniadas. Esses aspectos seriam mais adequadamente tratados se fosse possível o acesso a dados sobre a evolução da utilização dos recursos do SUS em diferentes períodos de tempo (por exemplo, uma série temporal das percentagens de ou privados, não têm como se basear em sistemas de armazenamento dos recursos pagos pelo governo, o que inviabiliza análises de séries históricas.

Os dados disponíveis permitem avaliar algumas das implicações da implantação do SUS na microrregião de Ponte Nova (via NOB91). Verifica-se que os hospitais conveniados passam a priorizar o investimento em convênios privados, diminuindo, gradativamente, os atendimentos aos pacientes do SUS. Com isso, emerge uma nova modalidade de seguros-saúde privados - os convênios exclusivos das unidades de saúde.

Quadro 2 - Modalidades de Convênios e Percentagens de Arrecadação por Unidades de Saúde Privadas. Microrregião de Saúde de Ponte Nova-MG. 1995

\begin{tabular}{|c|c|c|c|c|c|c|}
\hline \multicolumn{7}{|c|}{ Fontes de arrecadação/\% } \\
\hline \multirow[t]{2}{*}{ Instituição } & \multicolumn{6}{|l|}{ Local } \\
\hline & & $\begin{array}{l}\text { Plano } \\
\text { saúde } \\
\text { próprio }\end{array}$ & $\begin{array}{c}\text { Data } \\
\text { implantação } \\
\text { plano próprio }\end{array}$ & $\begin{array}{c}\text { Outros } \\
\text { convênios }\end{array}$ & SUS & Particulares \\
\hline $\begin{array}{l}\text { Hospital } \\
\text { N.S.D. }\end{array}$ & Ponte Nova & 23,0 & $\begin{array}{c}1 .{ }^{\circ} \mathrm{sem} / 1993 \\
\text { (PLAMAG) }\end{array}$ & 15,0 & 49,0 & 13,0 \\
\hline $\begin{array}{l}\text { Hospital } \\
\text { A.G.F. }\end{array}$ & Ponte Nova & 24,0 & $\begin{array}{r}1 .^{\circ} \text { sem./1992 } \\
\text { (Plano Família) }\end{array}$ & 15,0 & 47,0 & 14,0 \\
\hline Consultar & Ponte Nova & 100,0 & $1 .^{\circ}$ sem. $/ 1991$ & - & - & - \\
\hline $\begin{array}{l}\text { Hospital } \\
\text { I.S.G. }\end{array}$ & Guaraciaba & - & - & 3,0 & 95,0 & 2,0 \\
\hline $\begin{array}{l}\text { Hospital } \\
\text { N.S.L. }\end{array}$ & Alvinópolis & - & - & 6.0 & 84.0 & 10.0 \\
\hline
\end{tabular}

Fonte: Dados da pesquisa.

arrecadação dessas unidades de saúde, ano a ano). Embora fosse esse um aspecto a ser pesquisado, durante a coleta dos dados, nenhuma das instituições, públicas ou privadas, forneceu tais dados, alegando não possuí-los. A dificuldade de acesso aos dados das unidades de saúde foi destacada por Giovanella e Bahia (1995). Tais autores ressaltam que, no Brasil, a análise de custos e a comparação entre os recursos públicos utilizados pelos hospitais, sejam eles públicos
Especificamente, para a situação investigada, identificou-se a criação dos planos específicos dos hospitais, PlAMAG e Plano Família, como alternativas para os planos de saúde existentes, e a criação da Consultar, que é uma empresa privada de consultas médicas. Esses convênios possuem 15.047 titulares, sem considerar o número de dependentes e o de conveniados em nível empresarial. A situação se revela ainda mais grave, conforme descrito pelos diferentes atores 
entrevistados (usuários, membros dos Conselhos Municipais de Saúde, Diretores administrativos dos hospitais), pois, na prática, o que tem ocorrido é apenas a substituição do cliente do SUS pelo cliente do plano do hospital. Ou seja, a cada procedimento hospitalar realizado para os clientes dos planos privados é emitida uma guia de pagamento do SUS. É o sistema público de saúde subsidiando e alavancando o setor privado.

É importante ressaltar que o número de titulares desses convênios representa 30\% da população total do município onde foi implantada essa modalidade de seguro privado e $20 \%$ da população dos municípios amostrados nesse estudo.

Nota-se que esse processo de expulsão dos atores para os serviços privados de saúde ocorre justamente com aqueles indivíduos que mais se preocupam com a qualidade do produto e que, conseqüentemente, seriam os agentes mais ativos e criativos da "voz" (Hirschman, 1973). Esse processo de retirada da esfera do setor público dos segmentos da população com maior capacidade de vocalização de demandas pode levar ao risco de perpetuação do circuito de má qualidade: expulsão de setores organizados, baixa atividade reivindicatória, dificuldade de acesso, má qualidade (Faveret \& Oliveira, 1990).

Diante da incapacidade do setor público, em todos os sentidos, em atender às necessidades de saúde da população local, verifica-se que o discurso governamental, segundo o qual, ao deixar para o subsistema privado os segmentos de melhor nível de ren$\mathrm{da}$, abre-se espaço para que o Estado se capacite a atender mais eficientemente os setores sociais de menor poder aquisitivo, não se tem efetivado na prática (Faveret \& Oliveira, 1990). Pelo contrário, são justamente estes últimos setores sociais que têm encontrado mais dificuldades no acesso aos serviços de saúde.

O interessante é que isto não ocorre pela deficiência dos serviços em si. Como a maioria dos convênios privados não cobre os serviços de alto custo (tais como hemodiálise e oncologia), que ficam por conta do SUS, mesmo quando o paciente possui plano privado de saúde, conclui-se que o mérito da eficácia, da resolutibilidade e do acesso é muito mais político do que técnico ou financeiro. Na prática, não há falta de profissionais qualificados ou de tecnologia (Cohn, 1989).

Esses aspectos podem ser mais ilustrativos se se examinar o que ocorre com os atendimentos dos municípios em estudo (Quadro 2). Na medida em que o SUS deixa de ser um "bom cliente", ocorre uma ampliação da rede privada de saúde. Os dois hospitais do município criam convênios de saúde próprios, passando a responder por 23 e $24 \%$, respectivamente, do orçamento global dessas instituições. Além disso, identificou-se que essas instituições optaram, também, pela ampliação do número de convênios com empresas e com particulares. Um dos entrevistados, que ocupa o cargo de administrador hospitalar, comenta:

"A partir de 1991, passamos a investir nos convênios. Todos aqueles convênios que pagam tabela da AMB são muito mais interessantes que o SUS, nossa meta é reduzir os atendimentos do SUS ao minimo possivel. Uma prova de que a população está descrente do SUS é o fato de ter sido implantada, com sucesso, a empresa médica Consultar, que atende só em nível de consultas. Como o maior medo do paciente é precisar se internar pelo SUS, é claro que os planos dos bospitais iriam dar certo...".

Ao priorizar a política de investimento em convênios privados, esses hospitais que, até então, tinham $90 \%$ de seu orçamento provenientes de recursos do SUS reduziram, pelo menos em tese, esses recursos para menos de $50 \%$. Isso evidencia a dificuldade 
de acesso aos serviços de saúde do SUS pela população local. Mas, cabe observar que, na prática, as instituições privadas conveniadas reduziram apenas o acesso aos clientes do SUS, não os procedimentos (a arrecadação pelo SUS). Elas continuam a receber pelos atendimentos, pois emitem guias de atendimento para grande parte dos pacientes conveniados com os planos de saúde dos hospitais.

$\mathrm{Na}$ medida em que na regional de saúde em estudo não existe nenhum hospital ou serviço público estruturado que atenda em nível de urgência-emergência ambulatorial e de internação, constata-se que $40 \%$ da capacidade de atendimentos nessas áreas deixaram de estar disponíveis para os clientes que têm no SUS sua única alternativa. Como não ocorreu uma substituição dessa capacidade de atendimento pelo setor público, haveria, na verdade, uma tendência a reduzir o acesso aos serviços do SUS.

Nos municípios de menor porte a situação é diferente. Neles, a dependência dos recursos provenientes do SUS é quase total, pois o nível de renda da população é baixo. Entretanto, aqueles com maior poder aquisitivo optam por fazer convênio com os hospitais de Ponte Nova, em razão do maior número de especialistas e de equipamentos. Há ainda um outro agravante no que diz respeito à infra-estrutura dos serviços de saúde. O município de maior porte, por possuir maior variedade de especialistas e recursos tecnológicos, serve como referência para os demais da região sob estudo. Assim, a opção dessas unidades pelos planos privados de saúde agrava ainda mais a questão do acesso aos serviços de saúde pelas camadas da população que, por não possuírem recursos para se associarem às diferentes modalidades de seguros-saúde, têm, no SUS, sua única forma de assistência. Há ainda, como agravante, o fato de que esses hospitais têm emitido AIHs (Autorização de Internação Hospitalar) para os pacientes desses planos privados. Tal procedimento faz com que, mais uma vez, aquele indivíduo carente, que não possui recursos para pagar por um atendimento particular ou por um plano de saúde, não consiga ser atendido pelo sistema público.

Entre as causas que levaram os hospitais a implantar planos de saúde privados, os administradores hospitalares apontam a necessidade de garantir uma clientela fixa para comprar os serviços do hospital que remunerasse mais adequadamente, e em dia, os serviços prestados. Curiosamente, outra causa apontada, para além do cálculo econômico racional, foi a reorganização do atendimento provocada pelo SUS. Os entrevistados comentam que, na época do INAMPS, a população tinha direito à internação em quartos particulares, tendo apenas que complementar as diárias e os serviços médicos. O INAMPS pagava o serviço de hotelaria, as taxas do bloco cirúrgico, os medicamentos, parte das despesas médicas e de hospedagem. Com a implantação do SUS, ou o usuário paga por todos os serviços, ou se interna em enfermarias. Esses atores destacam que o SUS, pelo fato de só dar direito à internação em enfermarias e de dificultar cada vez mais as cirurgias eletivas, além das constantes filas nos serviços ambulatoriais e da baixa qualidade dos serviços oferecidos, não atende às expectativas mínimas de respeito e dignidade dos usuários. Aqueles que podem se retiram para o mercado. Aqueles que não o fazem, resignam-se. De acordo com reportagem da revista VEJA (1992:78):

"(..) o número de segurados de mais de 400 empresas dedicadas a prestar seguro ou assistência de saúde privada no Brasil cresce 30\% ao ano. Quase toda a classe média brasileira já está segurada e agora os planos começam a ser oferecidos a pessoas de renda mais baixa".

Essa tenclência pode ser evidenciada tanto pela criação desses seguros específicos por instituições de saúde, como é o caso dos dois 
hospitais de Ponte Nova, como pelos convênios coletivos, realizados por sindicatos $\mathrm{e}$ empresas, para todos os empregados.

Para conhecer adequadamente a tendência que o sistema de saúde local vem manifestando, deve-se prestar atenção às causas atribuídas pelos atores para a proliferação dos planos de saúde privados. De acordo com $45 \%$ dos entrevistados, a principal causa de fuga da população dos serviços públicos de saúde se refere à garantia de atendimento e de acesso aos serviços médicos, associado ao direito de escolha, tanto do médico como da instituição de saúde. Deve-se destacar, aqui, a questão da "impessoalização" do atendimento, que o usuário aponta como um dos pontos negativos do SUS. Questões puramente econômicas não são avaliadas: o mais importante para $45 \%$ desse contingente é "repersonalizar" a relação com o médico que o atende. Além disso, 30\% dos atores destacam a procura pela qualidade e resolutibilidade no atendimento, o que não é oferecido pelo SUS.

Outra causa determinante do crescimento dos planos de saúde privados estaria relacionada aos incentivos decorrentes das nãodecisões do próprio governo. O sucateamento do SUS alimenta a privatização, reduzindo a saúde pública aos carentes da população brasileira. Para $25 \%$ dos entrevistados, essa é a principal causa da tendência à privatização. Nota-se que atualmente a atenção se centra mais na questão do acesso e da cobertura, do quee resolubilidade.

Deve-se destacar ainda uma segunda forma de subsídio, por parte do governo, aos setores privados de saúde. De acordo com Pastore e Varella (1996), enquanto em 1988 os seguros de saúde privados davam cobertura a $10 \%$ da população (eram privilégio de 14 milhões de brasileiros), hoje atendem a cerca de 24\% (cerca de 35 milhões). Ainda segundo esses mesmos autores, tal incremento da medicina privada tem sido intencionalmente estimulado pelo governo:
"O governo tem tudo a ver com a explosão da medicina privada no Brasil. Todas as empresas cresceram com o auxilio de um subsídio indireto, da ordem de 2 milhões de dólares. Essa quantia equivale ao que o imposto de renda deixa de receber todos os anos, ao permitir que os gastos com planos de saúde e despesas médicas em geral sejam abatidos da declaração do imposto de renda. Sem contar que a maioria dessas empresas ainda usufruem de outra vantagem: são cadastradas como entidades filantrópicas, sem fins lucrativos, o que também as alivia de pagar sua parte no fisco" (Pastore \& Varella, 1996:54).

É interessante notar que - como a maioria dos seguros de saúde privados não cobre a medicina de alto custo - o paciente necessitado de serviços desta natureza é, na verdade, atendido pelo SUS. Segundo os entrevistados, esses serviços, em geral, são bem organizados e o acesso não é tão difícil como nos outros serviços rotineiros. Quando existe pagamento adequado e digno (medicina de alto custo) o SUS anda bem. Entretanto, a clientela que utiliza esses serviços é bem restrita. Para Giovanella e Bahia (1995:12), esse aspecto ilustra a inferência de que o governo deixa todos os espaços possíveis para que os planos de saúde privados possam evoluir naturalmente:

"É sempre bom lembrar que os planos de saúde prestam apenas assistência médica individual, não realizam ações de caráter coletivo, excluem da atenção uma série de doenças crônicas e endêmicas e não oferecem serviços qualificados para o atendimento de grandes emergências, atenção esta que tem sido suprida pelos serviços públicos".

A maioria dessas doenças (crônicas e endêmicas) poderiam ser solucionadas com ações de caráter preventivo e a um custo muito mais baixo (Mendes, 1992). Como a implantação do SUS não reverteu a lógica da 
produção dos serviços de saúde de caráter curativo, a tendência é a elevação progressiva dos custos com a atenção à saúde. O caso de pacientes que se submetem ao tratamento de hemodiálise pode ser tomado como exemplo. Geralmente, são pessoas com história de diabetes e/ou hipertensão que, quando submetidas a um acompanhamento médico preventivo, não chegam a necessitar de hemodiálise. Por outro lado, uma vez que se submetam a esse tipo de tratamento, necessitarão de cuidados permanentes (Giovanella \& Bahia, 1995). Chama atenção o fato de que, segundo dados da Secretaria de Estado de Saúde, de Minas Gerais, os gastos em serviços de hemodiálise, na regional de saúde sob estudo, é um dos mais altos do Estado.

Outro aspecto importante a ser ressaltado com relação aos seguros de saúde privados é que, pelo menos no caso dos planos de saúde dos hospitais das principais cidades da regional de saúde de Ponte Nova-MG, estes recebem do SUS por grande parte das internações e por outros atendimentos de pacientes dos planos. É o sistema público de saúde (SUS) "subsidiando" os seguros de saúde privados. A emissão de autorização de internação hospitalar (AIH) para os pacientes que se internam pelos planos de saúde está contribuindo, ainda mais, para a diminuição de vagas para os usuários que realmente não têm outra alternativa além do SUS.

Nesse sentido, os atores entrevistados apontam, como problemas do setor deficiências quantitativas na oferta dos serviços públicos de saúde, que se evidenciam na restrição do acesso aos atendimentos e nas deficiências qualitativas desses mesmos serviços de baixa resolubilidade e eficácia. Dessa forma, é de se supor que modalidades privadas de assistência médica continuarão a crescer. De acordo com Médici (1991:35):

"O pior é que a queda da qualidade e, acrescente-se, da quantidade dos serviços pres- tados pelo setor público e a inércia em proceder a esforços racionalizadores da máquina dos Estados e Municipios (em que pesem as exceções) colocam a possiblilidade de falência da atenção médica, mesmo para os segmentos mais pauperizados que, com a estrutura de distribuição de renda existente, longe estão de poder utilizar o modelo assistencial representado pela medicina de grupo no Brasil".

A questão da qualidade e da eficácia está presente tanto nas respostas dos 30\% que identificam a busca da qualidade e resolutibilidade nos atendimentos como causa de aumento da procura por planos privados, quanto nos $25 \%$ que apontam o próprio governo como responsável pela privatização da saúde. Segundo os últimos, o governo estaria deslocando deliberadamente para o subsistema privado os setores aptos para o mercado, ao sucatear o sistema público de saúde. O sucateamento é revelado nas tabelas irrisórias de pagamentos pelos serviços oferecidos, de um lado, e na incerteza quanto ao repasse de verbas, de outro. Isto sem se falar nos subsídios indiretos dos recursos públicos da saúde ao setor privado.

Por sua vez, o SUS, segundo reportagem do jornal Novo Rumo (1995:5) revela a possibilidade de uma alternativa inusitada:

"O Ministro da Saúde anunciou que o governo está estudando uma maneira de cobrar os serviços dos médicos e hospitais na rede pública e conveniada das pessoas que dispõem de sistemas alternativos de atendimento, como planos de saúde e cooperativas médicas".

A alternativa, apresentada pelo próprio governo, seria a legalização do pagamento "por fora", pelos pacientes ao profissional e à instituição, que já recebem os honorários do governo. Ou seja, o SUS pagaria as des- 
pesas de internação, tais como hotelaria, taxas cirúrgicas etc., e o usuário complementaria estas despesas médicas, sistema semelhante ao que ocorria no extinto INAMPS. Observese que a proposta do Ministro da Saúde é semelhante à do Banco Mundial (World Bank, 1987), segundo a qual a liberação do Estado de prover serviços para os ricos traria como conseqüência a alocação dos recursos no atendimento aos pobres. Também Teixeira (1994) destaca a importância da livre iniciativa na assistência à saúde. Para esse autor, a solução para o fracasso do SUS seria a parceria entre as empresas médicas de seguro de saúde e o setor público.

Essas propostas trazem o risco de gerar formas de atendimento seletivo nos hospitais do (SUS) e conveniados, dificultando, ainda mais, o acesso para os setores da população que não dispõem de planos privados de saúde (Misoczky, 1995). De acordo com Carvalho (1995:42-43), isso já vem ocorrendo na prática:

"(...) como já ocorre em dois grandes bospitais públicos do pais que já admitem o tratamento privado: o Instituto do Coração INCOR e o Hospital das Clínicas em Porto Alegre. Nesses hospitais, os serviços de saúde pública são divididos em dois: um para os que podem pagar e outro para os que não podem. Por exemplo, no INCOR, o ambulatório para atendimento do SUS fica no subsolo do edifício. A sala de espera tem poltronas de plástico e está sempre lotada. Sentados, alguns pacientes recebem soro ali mesmo. É o serviço dos pobres. O serviço para os que têm dinbeiro para pagar pelo atendimento fica no andar de cima. Na entrada, uma placa afasta os desavisados: 'Consultórios - Convênios e Particulares'. Suas poltronas são de couro e o piso tem carpete. Quem tem dinbeiro para pagar é atendido imediatamente, quem é cliente do SUS corre o risco de esperar até três meses".

\section{Considerações Finais}

As formas perversas de interação do sistema público de saúde com o mercado, apontadas anteriormente, parecem evidenciar um retrocesso na forma de custeio da saúde no país. Algo semelhante já havia acontecido com os Institutos de Aposentadorias e Pensões (IAPS) nos anos 30, quando as categorias profissionais optaram por esquemas próprios de concessão pela assistência médica exclusiva, mediante convênios coletivos, via sindicatos ou empresas (Oliveira \& Teixeira, 1989).

Hoje, verifica-se um retorno à fragmentação do sistema, com o agravante de que atualmente os trabalhadores inseridos no mercado formal de trabalho (com carteira assinada), pela ineficiência do setor público em prover serviços de saúde em quantidade e qualidade satisfatórias, pagam duplamente pela possibilidade de acesso aos serviços de saúde (pelo serviço público e pelo privado). Têm-se, de um lado, a obrigatoriedade de contribuição previdenciária e, de outro, o pagamento de seguros privados de saúde, sem, ao final, ter-se a garantia de um atendimento satisfatório.

$\mathrm{Na}$ prática, a forma desorganizada de o governo implementar a Reforma Sanitária manifesta no crescente "desfinanciamento" do setor, má administração dos recursos, ineficácia gerencial e a não-regulamentação dos planos privados - sugere que se encontra em curso um processo de privatização da saúde que, num primeiro momento, não foi resultado de ações explícitas do governo, mas que se realizou e se realiza por meio de nãodecisões. O governo parece ter adotado a postura de deixar o setor à própria sorte. Como resultado, constata-se que, concomitantemente ao processo de sucateamento da saúde pública, se expandem as diferentes modalidades de seguros privados de saúde.

O interessante é que na saúde ocorre algo inusitado. Conforme demonstra este estudo, o sistema público de saúde, mesmo 
para aqueles que optaram pela saída para o mercado, tem sido duplamente utilizado para subsidiar o setor privado. Primeiro, quando os planos de saúde dos hospitais emitem AIHs para os pacientes conveniados e depois, quando justamente naqueles procedimentos de saúde mais onerosos, a saúde pública continua a ser a única alternativa. Isso ocorre porque a maioria dos planos de saúde (90\%) não cobrem tratamentos de doenças crônico-degenerativas e infecto-contagiosas, doenças preexistentes ou atendimento de urgência-emergência. Caso necessite desse tipo de assistência, o cliente geralmente recorre ao SUS, o que faz com que, na saúde, seja praticamente impossivel ocorrer a "saída" total para o setor privado (Hirschman, 1973).

A intenção dos idealizadores da Reforma Sanitária era de que o SUS tivesse implicações redistributivas. Embora seu arcabouço institucional, fundado nos princípios de universalidade e eqüidade do atendimento, represente claramente essa intençâo redistributiva, a crise do sistema, na medida em que não deixa o que redistribuir, tem produzido efeitos altamente regressivos.

Como se analisou extensamente no decorrer deste estudo, os avanços jurídico-legais obtidos na área da saúde não se têm traduzido em melhoria dos serviços públicos de saúde ou em ampliação do acesso e da cobertura desses serviços. Ao contrário, o que se observa é a queda na qualidade e o incremento de mecanismos que visam a dificultar

\section{Referências bibliográficas}

BABBIE, E. (1983) - The Practice of Social

Research. 3rd. Belmont: Wadsworth Publishing Company.

CARVAlHO, G.C. (1995) - O SUS dos Pobres e o SUS dos Ricos. Súmula - Radis, Fiocruz, p. 5.

COHN, A. (1989) - Caminhos da Reforma Sanitária.

Lua Nova 19:123-140.

FAVERET, P. \& OLIVEIRA, P. (1990) - A Universa-
- acesso da população a esses serviços. Aparentemente, depara-se com a segunda fase do projeto de privatização da saúde em curso. No momento atual, quando o setor privado cresce vertiginosamente e às custas de recursos públicos, o governo já se posiciona mais claramente e inicia investidas, agora explícitas, contra o SUS.

A nova política de saúde pretendia, ao transferir decisões sobre alocação de recursos e procedimentos para a esfera local, eliminar as irracionalidades produzidas pela gestão centralizada, atendendo de forma mais diferenciada e eficaz às necessidades de cada cidadão em seu município. Paradoxalmente, entretanto, a análise do funcionamento do sistema nos municípios amostrados mostrou que a fragmentação dos mecanismos de decisões, ao invés de resultar em ação, provocou paralisia decisória.

Por fim, a análise dos dilemas que atravessa a implementação da reforma do setor saúde, nos moldes propostos pelo movimento da Reforma Sanitária, leva a concluir que, a permanecerem as condições atuais, o cenário futuro do setor será de desagregação do SUS em seus princípios democratizantes de universalização, de eqüidade e integralidade. Com o agravante de que, no jogo do "salvese quem puder" (O'Donnell, 1993) instituído na saúde, a fuga para o mercado longe está de constituir alternativa satisfatória, tanto para os usuários, quanto para os profissionais de saúde e a sociedade em geral.

lizaçào Excludente: Reflexões sobre as Tendências do Sistema de Saúde. Planejamento $e$ Politicas Públicas 3(1):139-152.

GIOVANELlA, L.\& BAHIA, L. (1995) - Saúde: O Poder da Atenção Pública. Saúde em Debate 47:8-13.

HIRSCHMAN, A. (1973) - Saida, Voz e Lealdade. São Paulo: Perspectiva. 
JELIN, E. (1993) - Construir a Cidadania: Uma Visão desde Baixo. Lua Nova 33:40-57.

MÉDICI, A. (1991) - A Medicina de Grupo no Brasil. Série Desenvolvimento de Politicas de Saúde 1. Washington: OPAS/OMS.

MENDES, E.V. (1992) - O Sistema Único de Saúde e o Papel das Secretarias Estaduais de Saúde. Cadernos da Conferência Nacional de Saúde 2:101-117

MISOCZKY, M.C. (1995) - A Agenda para Reformas do Banco Mundial e a Política de Saúde: Algumas Notas para a Reflexão. Saúde em Debate 47:4-7.

NOVO RUMO (Jornal) (1995) - O SUS Ameaçado de Intervenção, ediçào de julho, p. 5.

O'DONNELL, G. (1993) - Sobre o Estado, a Democratização e Alguns Problemas Conceituais: Uma Visão Latino-americana, com uma Rápida Olha- da em alguns Países Pós-comunistas. Novos Estudos 36:123-145.

OLIVEIRA, J. \& TEIXEIRA, S.F. (1989) (Im)Previdência Social, 60 Anos de História da Previdência no Brasil. Petrópolis: Vozes.

PASTORE, K. \& VARELLA, F. (1996) - A Medicina da Ilusão. Veja, edição de 8 de maio.

TEIXEIRA, I. (1994) - Saúde: O Poder da Privatização. Conjuntura Econômica 1:28-33.

VEJA (Revista) - ... A Saúde a Crédito. Edição de 25 de novembro, p.78.

WARWICK, D. \& LININGER, C. (1975) - The Sample Survey: Theory and Practice. Nova York: MacGraw-Hill Books.

WORLD BANK (1987) - Financing Health Services in Developing Countries: An agenda for Reform. Washington: The World Bank. 